\title{
Optical Safety Assessment of a Near-Infrared Brain-Computer Interface
}

\author{
Christopher J Soraghan ${ }^{1,3}$, Tomás E Ward ${ }^{2}$, Fiachra Matthews ${ }^{3,4}$, \\ Charles Markham ${ }^{3}$
}

\author{
${ }^{1}$ Department of Experimental Physics \\ National University of Ireland, Maynooth \\ ${ }^{3}$ Department of Computer Science \\ National University of Ireland, Maynooth
}

\author{
${ }^{2}$ Department of Electronic Engineering \\ National University of Ireland, Maynooth \\ ${ }^{4}$ Hamilton Institute, \\ National University of Ireland, Maynooth
}

email : christopher.j.soraghan@nuim.ie

\begin{abstract}
This paper describes a safety assessment study of near-infrared sources used in an optical brain-computer interface (BCI). The measurement elements of an optical BCI consist of sets of optical sources and detectors. Our current system utilises sources which comprise of dual wavelength light emitting diodes (LED) at $760 \mathrm{~nm}$ and $880 \mathrm{~nm}$. An optical analysis demonstrated that NIR radiation is a negligible source of heating in this case. LED heat conduction however is a major source of heating, and LEDs, though much safer than laser diodes, have been known to cause burns if improperly used. We describe a procedure by which we measure the heat conduction effect of LEDs. We show that the LED systems used in our current generation BCI produce safe levels of thermal energy and are within published safety levels.
\end{abstract}

Keywords - Brain-Computer Interfaces, Light Emitting Diodes, Heating effects, Near-Infrared Spectroscopy.

\section{INTRODUCTION}

In this paper we assess the safety of a novel brain-computer interfacing system which utilises incoherent near-infrared light to interrogate brain state.

\subsection{Brain-Computer Interfaces}

A brain-computer interface (BCI) is a device which allows a user's intentions to be monitored independent of communication via conventional nervous pathways. Using such a device a user can communicate and interact with the outside world through thought process alone. Since there is no involvement of the peripheral nervous system such techniques may for example compensate for damaged pathways allowing communication for the most profoundly disabled in society.

The paradigm for current systems is straightforward relying on basic physiological measurement typically electrical in nature although other modalities have been used; the optical system used in this study represents such an alternative system. Crucial to any BCI system is a means through which volitionally induced thought patterns can be distinguished from ongoing brain activity.
Any measurement modality which allows such a pattern in the measurand to be discerned is an excellent candidate to be used as part of a BCI. Communication is enriched subsequently through the use of simple sequential option scanning usually in software. Typically such software takes the form of a spelling device or other such aid [1].

An optical BCI (OBCI) uses tissue interrogation with light rather than measurement of electrical fields to determine volitionally-induced brain activity [2]. In this paper we utilise a custom OBCI which employs near-infrared (NIR) light to determine dynamic measures of neurovascular coupling non-invasively in the brain. Local neurovascular coupling variation is correlated with brain activity facilitating a coarse yet robust method for brain-computer interfacing $[3,4]$.

\subsection{The NIRS Measurement Principle}

The basic measurement principle is based on the optical window which exists in biological tissue (approximately 650-900nm). In this range photons can penetrate the outer tissues of the human head, including the cranium and the various meninges 
(dura matter, arachnoid, and pia matter) and fluids surrounding the brain. Such photons are highly scattered. As the photons diffuse a number are absorbed by molecules (termed chromophores) while others continue to be scattered following a random walk path through the medium. Consequently many of these photons are back-scattered from the tissue and exit the surface some centimetres from their original entry point. The path history of a subset of the photons which enter at the source location and exit at a fixed measurement location constitute an optical interrogation path through the tissue as shown in Fig. 1.

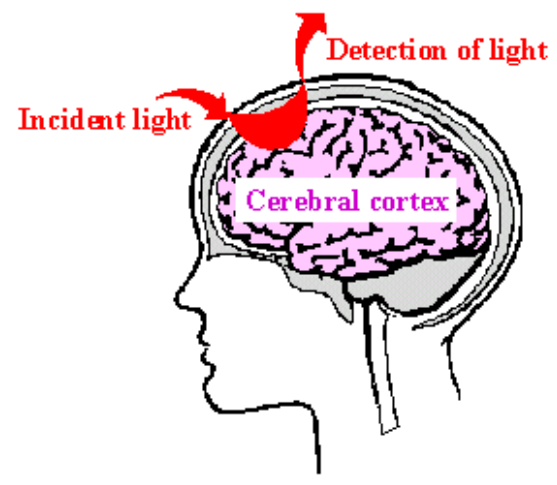

Fig. 1: Illustrative optical interrogation path during nearinfrared sensing.

The actual amount of back-reflected photons detected depends on the optical properties of the tissue and will change as concentrations of the various absorbers vary. This can be quantified to some extent using a modified version of the Beer Lambert Law.

$$
A=\log _{10} \frac{I_{o}}{I}=\alpha c L B+G
$$

where $A$ is attenuation, $I_{o}$ is incident light intensity $(m W), I$ is transmitted light intensity $(m W), \alpha$ is the specific extinction coefficient $\left(\mathrm{mol}^{-1} \mathrm{~m}^{-1}\right), c$ is the concentration of the absorber (mol), $L$ is the distance between the source and detector $(\mathrm{m}), B$ is the differential pathlength factor and $G$ is a term to account for scattering losses.

One such absorber is the haemoglobin molecule which shows different absorption characteristics depending on whether it is in reduced or oxygenated form (Fig. 2). Consequently through measurement of $A$ at a number of wavelengths simultaneously it is possible to resolve haemoglobin into its two dominant states. This is key. During concerted cortical activity a neurovascular process occurs whereby changes occur in cerebral blood flow, volume and metabolic rate of oxygen consumption.
The relationship between these variables is termed neurovascular coupling (NC).

It is known that NC undergoes localized changes during mental activity hence its potential utility as a BCI modality. Oxy-haemoglobin (HbO) and deoxyhaemoglobin (HbR) can be related to $\mathrm{NC}$ as discussed in $[5,6]$. An NC event during mental activation will affect the concentrations of oxyhaemoglobin and deoxy-haemoglobin as illustrated in Fig. 3 leading to transient changes in the respective states of haemoglobin. Such a response is a signature of mental activation and can be produced volitionally through appropriate mental activity. This is the basis of the optical or more accurately the near-infrared spectroscopy based BCI used here.

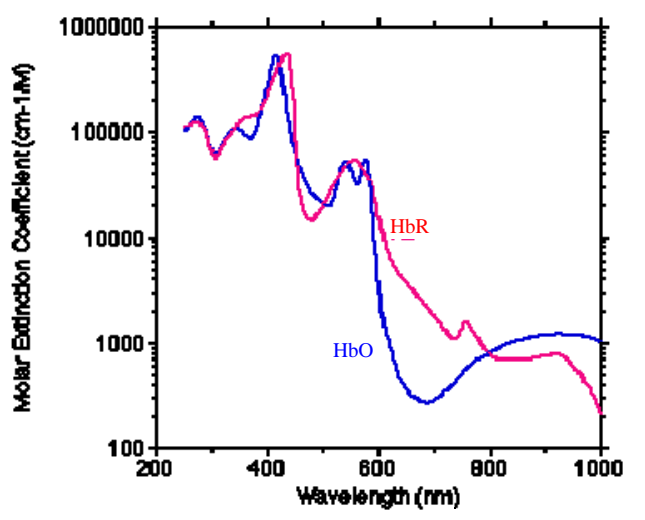

Fig. 2: Optical Absorption of Haemoglobin.

We have developed a robust, inexpensive, light emitting diode (LED) based, multi-channel optical system capable of topographic imaging for such purposes. The main elements of the cwNIRS system are the triple wavelength LED light sources (Opto Diode Corp. APT0101 - 760nm, 800nm, 880nm) with driving electronics (only 2 wavelengths are configured 760nm, 880nm), avalanche photodiode (APD) detectors (Hamamatsu C5460-01), coupling optics, mechanical harnessing to hold 'optets' (we refer to a number of sets of sources with a single detection site as an 'optet', as illustrated in Fig. 4) in place, and the data acquisition and processing unit.

The main control workstation is run on a LabView ${ }^{\circledR}$ platform which fully integrates every aspect of data processing and control. This graphical programming software application interfaces with a National Instruments PXI1033 chassis which houses two 4-channel ADC cards (PXI4462; 24-bit, 204kHz simultaneous sampling), and a single multifunction DAC (PXI6723) with 32-analogue output channels for driving the LED lights.

A custom-made stable voltage-to-current converter receives these biased carrier signals for driving the LEDs (applied perpendicularly to the scalp surface) which then irradiate the tissue. The back-scattered photons are then collected via fibre optic bundles (also applied perpendicularly to the 
scalp, 3-4 centimeters from the irradiated site) which guide the photons to the active areas of the APDs. The APDs transducing the photons to current then convert the current to voltage levels which are read directly by the ADC cards (using onboard analogue gain and anti-alias filtering) housed in the NI chassis. Data synchronization is keep robust via trigger lines running through the back pane of the chassis also allowing both analogue generation and acquisition to run on the same $10 \mathrm{MHz}$ clock.

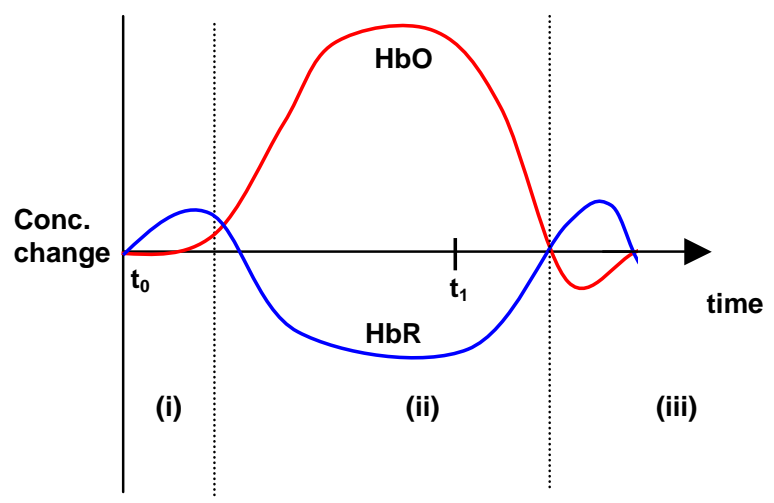

Fig. 3: Schematic depiction of the typical haemodynamic response. Stimulus occurs at time $t=t 0$ and ceases at $\mathrm{t}=\mathrm{t}_{1}$. There is (i) an initial increase in HbR, followed by (ii) decrease in HbR and increase in HbO. (iii) When the stimulus ends the $\mathrm{HbR}$ and $\mathrm{HbO}$ levels return to baseline.

\subsection{Safety Risk}

The chief hazard associated with the use of an optical brain-computer interface is tissue damage as a result of the use of optical sources. While the measurement is non-invasive (i.e. there is no breaking of the skin) it is not a passive one. Unlike scalp electroencephalography (EEG) which measures the inherent electrodynamics associated with neural activity, optical measurement as described here requires an external controlled source of photons with which to interrogate the tissue. The application of this optical flux is an inherent part of the instrumentation and must be carefully designed so as to ensure safety. A suitable stable source of NIR light for these applications could be laser diodes however the coherent nature of the light generated by such devices presents a significant risk of eye damage. A better solution is offered by incoherent sources such as LEDs. The short transport scattering length (the distance over which a collimated beam effectively becomes diffuse) at these wavelengths mean that the laser and LED solution are indistinguishable after a few millimetres penetration in the tissue.

Using LEDs, the principle risk is skin damage as a consequence of heating effects. The heating effect is determined by two mechanisms: radiated NIR light energy absorbed by the skin and thermal conducted energy which arises in the $p$ - $n$ junction during operation. The OBCI uses a significant number of LEDs for full operation therefore a safety analysis is important. The next section describes an assessment of the optical systems used in the OBCI under both irradiance and thermal analyses. Section III discusses the final findings of the reported assessments while Section IV concludes the work.

\section{SAFETY ANALYSIS}

As part of the initial safety assessment study some consideration is given to the numbers and types of light sources which will be applied to the head of the subject. Consideration is given then to their relative positions and absolute placement. A useful guiding principle is to use no more instrumentation or signal than necessary. Once established, analysis proceeds at the level of individual sources.

\subsection{Probe Patterns}

We use a number of channels in order to build up an "image" of cerebral tissue oxygenation. This is done dynamically such that changes resulting from different brain activities can be determined. A basic BCI experimental set-up might use left-arm imagined movement versus right-arm imagined movement as the basis for a communication strategy. This requires the application of two optets, each one situated over the appropriate parts of the motor cortex. This is illustrated in Fig. 4. The sets of mental states to be distinguished can be further enriched through monitoring left and right frontal cortex activity such that linguistic and arithmetic tasks can be separated. This would involve the application of two more optets. List 1 details typical activation strategies.

\section{Actual movement of a particular limb \\ 2. Imagined movement of a particular limb}

\section{Imagined tongue movement}

\section{Engagement in mental arithmetic}

\section{Engagement in language task}

List 1: Typical mental activation tasks used for optical BCI experiments. Each task requires a separate optet.

The optet design has been arrived at experimentally and uses seven dual wavelength LEDs (again, only 2 of the three wavelengths in the triple dye LED are ever configured) in contact with the scalp. The measured exit site for the photonic flux is a fibre optic bundle which takes light to the measurement system. The seven LEDs are a potential source of heat. The current optet design (see Fig. 4) positions each source radially $(3 \mathrm{~cm})$ such that there is minimal 
overlap between flux patterns (irradiance minimisation) and sufficient scalp between sites to reduce thermal focus. This is supported by the findings by Ito et al [7] where they express empirically that sources may be treated independently for inter-optode spacings greater than a few millimetres. Fig. 5 shows an optet together with the LED sites, detector, and the resultant optical density reconstructions.

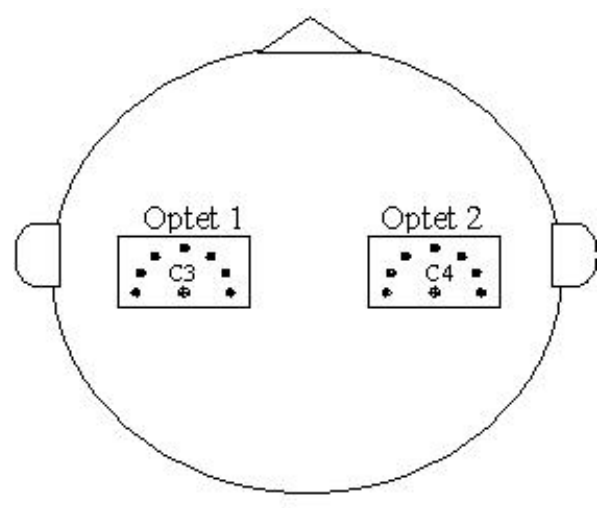

Fig. 4: Two optets placed over C3 (principally right hand imagery) and C4 (principally left hand imagery), located over the motor cortex to monitor contralateral motor imagery.
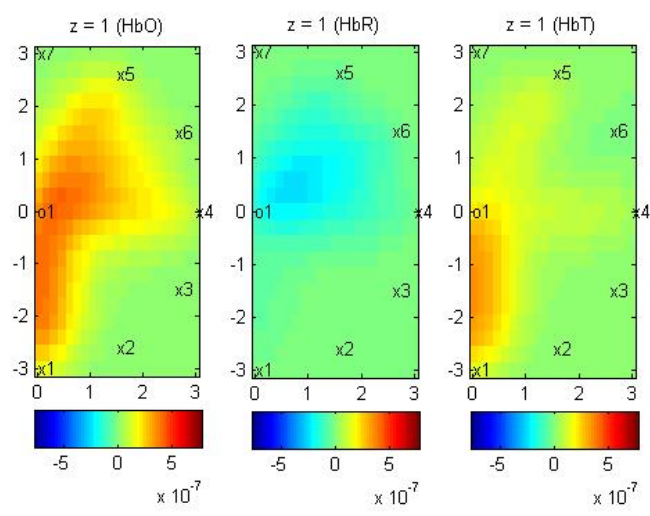

Fig. 5: Reconstruction of $\mathrm{HbO}, \mathrm{HbR}$, and $\mathrm{HbT}$ (total haemoglobin) optical densities. LEDs are located at $x 1$ to $x 7$. o1 designates the measurement point. $\mathrm{X}$ - and $\mathrm{Y}$-axis are in units of $\mathrm{cm}$.

\subsection{LED Radiated Energy Assessment}

Irradiance of tissue will cause some heating effects. This effect is dominated by absorption of the light by water. This heating effect increases towards the longer wavelengths of the NIR spectrum. The temperature increase as a result of radiated energy has been shown by Ito et al [7] to be less than $0.5^{\circ} \mathrm{C}$ for a typical continuous wave near-infrared sensing system. In addition, tissue rapidly dissipates heat as a function of tissue depth, thus ensuring deeper tissues are not adversely affected. Various compensatory reflexes also occur in the presence of increased temperature effects when flagged by skin thermoreceptors. A more conservative assessment based on classifying the LED as a laser is as follows based on the argument presented in [6]:

It is known [8] that $>95 \%$ of the photonic energy in the relevant spectrum interacts with the epidermis in the vicinity of the light source. For such biophotonic interactions the International Commission on Non-Ionizing Radiation Protection (ICNIRP) and the International Electrotechnical Commission (IEC) (British Standards 1994; ICNIRP Guidelines 2000) specify maximum safe exposure duration limits which should be adhered to.

Unlike lasers the optical radiation of LEDs are incoherent and in terms of regulations are categorised between incoherent broadband and coherent laser sources (ICNIRP Guidelines 1997). The ICNIRP recommends that the safety evaluations and related measurements of LEDs should adhere to the guidelines for incoherent sources. However the IEC includes LEDs in its safety guidelines for laser products (British Standards 1994). We adopt this position. Accordingly the IEC safety standards (British Standards 1994), specify that for a coherent light source in the $700 \mathrm{~nm}$ to $1400 \mathrm{~nm}$ range the following Maximum Permissible Exposure (MPE) of skin, for an exposure time $\mathrm{t}$ where $10^{3} \mathrm{~s}<\mathrm{t}<3 \times 10^{4} \mathrm{~s}$ is:

$$
M P E=2000 \cdot 10^{0.002(\lambda-700)} \quad\left[\mathrm{W} / \mathrm{m}^{2}\right]
$$

Therefore a laser operating at $880 \mathrm{~nm}$ under these conditions has a MPE of $4.6 \mathrm{~mW} / \mathrm{mm}^{2}$.

Conservatively considering LEDs as lasers we can calculate as follows:

Two LEDs at different wavelengths are required at each point source. One LED with a spectral peak at $760 \mathrm{~nm}$, the other at $880 \mathrm{~nm}$. These LEDs have typical output powers of $5 \mathrm{~mW}$ and $17 \mathrm{~mW}$ respectively and are housed in a TO-46 packaging. Connecting the LEDs directly to the scalp delivers light to a surface area of approximately $20 \mathrm{~mm}^{2}$. Thus when the $760 \mathrm{~nm}$ and $880 \mathrm{~nm}$ LEDs are on continuously they provide irradiance of $0.25 \mathrm{~mW} / \mathrm{mm}^{2}$ and $0.866 \mathrm{~mW} / \mathrm{mm}^{2}$ respectively, when both LEDs are switched on simultaneously the combined light output still remains well below the MPE for coherent light in this wavelength range. Furthermore for signal identification purposes the sources are sinusoid-modulated yielding a further reduction in irradiance (approximately 50\%). This yields very low intensity signals much less than that of ordinary sunlight experienced at the Earth's surface (approximately $1 \mathrm{~mW} / \mathrm{mm}^{2}$ [7]) which is more efficiently absorbed than the near-infrared light used here [9]. In a study examining the heating effects in skin during near-infrared spectroscopic measurements the maximum temperature rise in the human forearm was measured to be $7.93 \times 10^{-6}$ 
${ }^{\circ} \mathrm{C} / \mathrm{mW} / \mathrm{mm}^{2}$ at $0.5 \mathrm{~mm}$ below the skin's surface. At greater depths the recorded temperature changes were even smaller [9]. From this we can conclude that the optical powers as harnessed for NIRS can be considered safe from an irradiance standpoint given that skin temperature is normally around $31^{\circ} \mathrm{C}$, and tissue damage occurs only above $41^{\circ} \mathrm{C}$ [9].

Such analysis does not consider the thermal effect associated with the diode semiconductor junction. This is explored next.

\subsection{Thermal effect of LED in direct contact} with skin.

More recently it has been reported in the literature [10] that a far more significant source of heating often neglected is simply due to the current flow across the semiconductor junction. In such cases the LED simply heats up as it conducts current. For a DC driven LED this can heat up the LED significantly such that a superficial burn is possible [11]. The measurement system used was the same as has been reported in Section I (1.2). Only one optet was used in all the following experiments which comprised of 7 LEDs and one detector. Velcro straps were used in each experiment to fasten the optet to the subject/model/phantom. The following optet environments were investigated:

a) A simplified optical phantom over 120 minutes.

b) Human Head (over the primary motor cortex), duration 40 minutes.

c) Polystyrene Model Human Head (models extreme temperature conduction case polystyrene is a poor heat conductor), duration 40 minutes.

In all cases the LEDs were driven in normal operation mode. Each LED was modulated between $1-20 \mathrm{kHz}$ with a maximum current of $100 \mathrm{~mA}$ $(\sim 160 \mathrm{~mW})$. Again, the LEDs used at $760 \mathrm{~nm}$ and $880 \mathrm{~nm}$ delivered output powers of $5 \mathrm{~mW}$ and $17 \mathrm{~mW}$ respectively. The resultant temperature profiles are given in Fig. 6, 7 and 8.

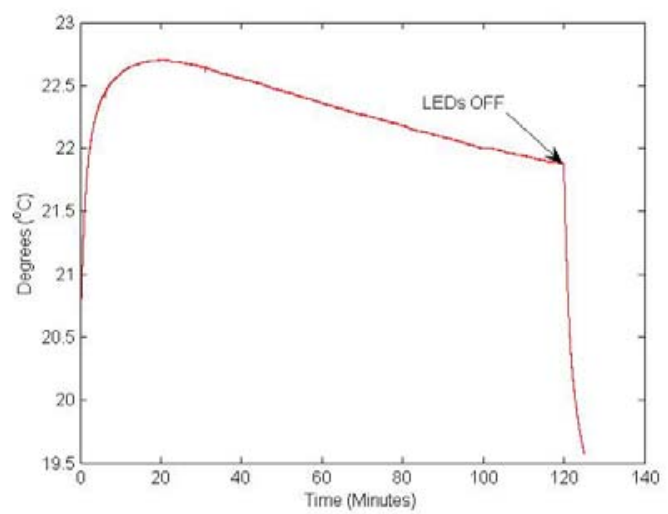

Fig. 6: Temperature Profile using a simplified optical phantom.
For Fig. 6, the temperature sensor (LM35) was in contact with the LED (2 wavelengths emitting light) and a plastic container using the same harness as used to fasten the LEDs on the head during a normal experiment on humans. The temperature reached a maximum of $\sim 22.7{ }^{\circ} \mathrm{C}$ after $20 \mathrm{mins}$ and did not exceed this over the 2 hours period. The temperature increase was approximately $3-4^{\circ} \mathrm{C}$ maximum from the recorded room temperature of $19^{\circ} \mathrm{C}$. The ambient temperature was $19.5^{\circ} \mathrm{C}$ in this case showing a maximum heating of $3^{\circ} \mathrm{C}$.

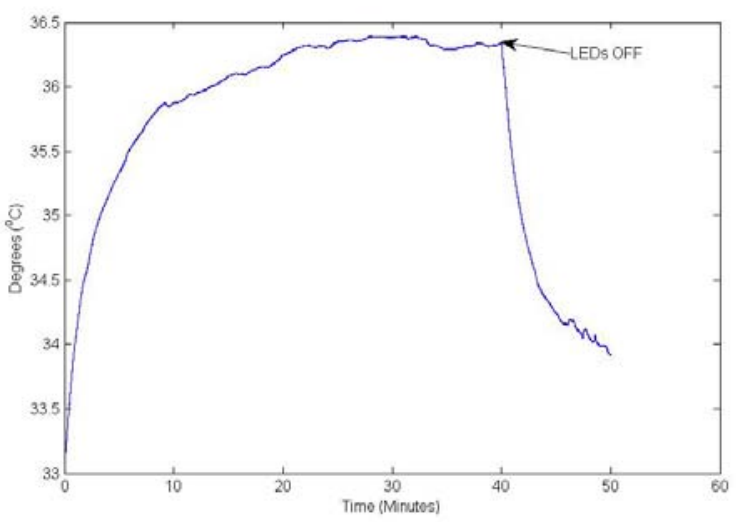

Fig. 7: Temperature Profile on Human Head.

Fig. 7 shows a recording over 40 minutes of the temperature change of the LED with 2 wavelengths emitting light. The temperature sensor (LM35) was in contact with the LED and a human subjects head again using the same harness as used to fasten the LEDs on the head during a normal experiment. The temperature reached a maximum of $\sim 36.4^{\circ} \mathrm{C}$ after 30 mins. The temperature increase was approximately $3-4^{\circ} \mathrm{C}$ maximum from the recorded scalp temperature of $33^{\circ} \mathrm{C}$.

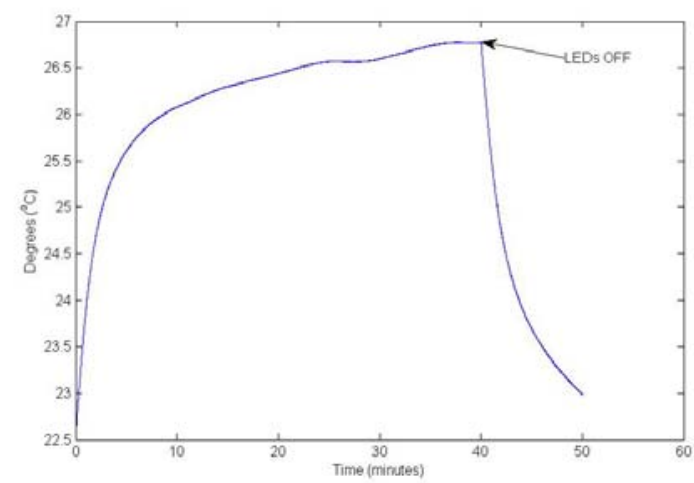

Fig. 8: Temperature Profile on Polystyrene Model of Human Head (models extreme temperature conduction case - polystyrene poor heat conductor).

Fig. 8 shows a recording over 40 minutes of the temperature change of the LED with 2 wavelengths emitting light. The temperature sensor (LM35) was in contact with the LED and a polystyrene head model using the same harness as 
used to fasten the LEDs on the human head during a normal experiment. The temperature reaches a maximum of $\sim 26.75{ }^{\circ} \mathrm{C}$ after 38mins. The temperature increase was approximately $4-5{ }^{\circ} \mathrm{C}$ maximum from the polystyrene head model temperature of $22{ }^{\circ} \mathrm{C}$. Again, it should be noted that the polystyrene head model was to demonstrate the worst-case scenario for conduction. Indeed it also lacks the thermoregulatory reflex abilities of a human head.

\section{DISCUSSION}

The temperature at which tissue damage occurs is $41^{\circ} \mathrm{C}$ as mentioned earlier. We have shown through a survey of the literature and worst-case calculation that the irradiance-induced thermal increase is essentially negligible for the optical sources we have been using. The instantaneous irradiance can be further reduced through the modulation of the LEDs rather than a DC operation. A further enhancement suggested in [10] is the use of a quadrature phase modulation system. This has been incorporated into the design of the LED driving element of the OBCI reported upon here.

In the case of thermal changes induced as a result of the heating of the semiconductor junction we have shown that our system presents no risk of skin damage. As we have demonstrated, our maximum irradiance, modulation strategies, peak powers, and system geometries all conform to the suggested safety guidelines being reported. A series of experiments carried out to confirm this hypothesis all verify that the level of induced temperature increase never exceeds $37^{\circ} \mathrm{C}$ in the case of the human head, and never increases by more than $5^{\circ} \mathrm{C}$ in the worst-case.

Thus, we have tested our system and in no case does the temperature increase above $41^{\circ} \mathrm{C}$ under the optical head.

\section{CONCLUSION}

The combined heating effect is important and the experiments described demonstrate how an assessment is possible of the thermal environment of the optical set-up used for a NIRS-BCI. In the case of the multi-channel optical BCI investigated here, it has been demonstrated that the instrument is thermally safe and can be considered a safe, noninvasive, non-ionising means of interrogating mental states for the purpose of a neural prosthesis.

\section{ACKNOWLEDGEMENTS}

This work is funded by Science Foundation Ireland Grant Number SFI/05/RFP/ENG0089 and is supported under the National Development Plan.

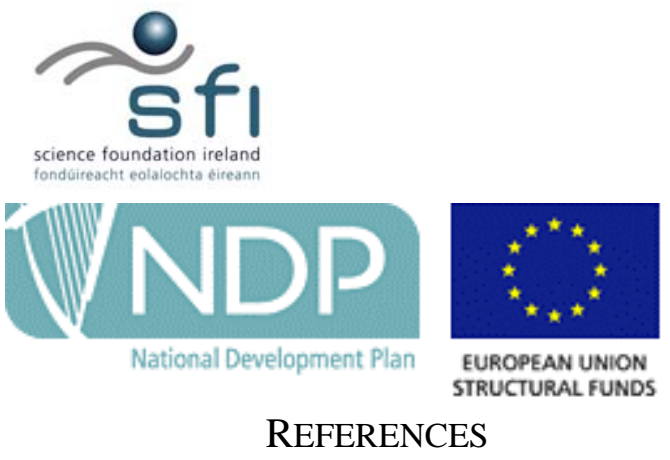

[1] Schalk, G., D. J. McFarland, Hinterberger, T., Birbaumer, N., Wolpaw, J. R. (2004). "BCI2000: a general-purpose brain-computer interface (BCI) system." IEEE Trans Biomed Eng 51(6): 1034-43.

[2] Matthews, F., Pearlmutter, B.A., Ward, T.E., Soraghan, C., Markham, C, "Hemodynamics for Brain-Computer Interfaces" (2008), IEEE Signal Processing Magazine, 25(1), pp. 87-94

[3]. S.M. Coyle, T.E. Ward, C. Markham, "Brain-computer interface using a simplified functional near-infrared spectroscopy system." (2007), Journal of Neural Engineering 4(3): 219-226.

[4] Ward, T.E., Soraghan, C.J., Matthews, F., Markham, C., "A Concept for Extending the Applicability of Constraint-Induced Movement Therapy through Motor Cortex Activity Feedback Using a Neural Prosthesis" (2007). Computational Intelligence and Neuroscience, vol. 2007, Article ID 51363, 9 pages, 2007. doi:10.1155/2007/51363

[5] Wolf M, Wolf U, Toronov V, Michalos A, Paunescu LA, Choi JH, Gratton E. "Different time evolution of oxyhemoglobin and deoxyhemoglobin concentration changes in the visual and motor cortices during functional stimulation: a near-infrared spectroscopy study." Neuroimage. 2002 Jul;16(3 Pt 1):704-12.

[6] S.M. Coyle, "Near-Infrared Spectroscopy for Brain Computer Interfacing," PhD thesis, National University of Ireland, Maynooth, 2005.

[7] Ito Y, Kennan RP, Watanabe E, Koizumi H: Assessment of heating effects in skin during continuous wave near infrared spectroscopy. Journal of Biomedical Optics 2000, 5(4):383-90.

[8] Strangman, G., D. Boas and J. P. Sutton: Non-Invasive Neuroimaging Using Near-Infrared Light. Biological Psychiatry 2002.52: 679-693.

[9] Sliney DH, Wolbarsht M: Safety With Lasers and Other Optical Sources: A Comprehensive Handbook. New York: Plenum Pub Corp; 1980.

[10] Bozkurt A, Onaral B: Safety assessment of near infrared light emitting diodes for diffuse optical measurements, Biomed Eng Online. 2004; 3: 9. Published online 2004 March 22. doi: 10.1186/1475-925X-3-9.

[11] Murphy KG, Secunda JA, Rockoff MA: Severe burns from a pulse oximeter. Anesthesiology 1990, 73(2):350-2. 